\title{
Photosensory Function, Reception and Response in Human Skin
}

\author{
David John Mackay Smith ${ }^{1,2, *}$ \\ ${ }^{1}$ Clinician, SunDoctors Skin Cancer Clinics Noosaville, Queensland, Australia \\ ${ }^{2}$ Senior lecturer in the Medical department, Queensland University, Australia
}

*Corresponding author: David John Mackay Smith, Clinician, SunDoctors Skin Cancer Clinics Noosaville, Queensland, Australia, E-mail: djmsmith8@ bigpond.com

Received: 21 Jun, 2021 | Accepted: 19 Jul, 2021 | Published: 26 Jul, 2021

Citation: Smith DJM (2021) Photosensory Function, Reception and Response in Human Skin. J Clin Cosmet Dermatol 5(2): dx.doi. org/10.16966/2576-2826.164

Copyright: (C) 2021 Smith DJM. This is an open-access article distributed under the terms of the Creative Commons Attribution License, which permits unrestricted use, distribution, and reproduction in any medium, provided the original author and source are credited.

\begin{abstract} of the organism as a whole. like melanoma.

\section{Human Response to the Threat of Ultraviolet Light Exposure}

Human skin has a photosensory system comprised of several opsins, light-sensitive proteins, and their signalling pathways as peripheral sensors and effectors mainly orchestrated through the melanocyte but also involving keratinocytes, fibroblasts and hair follicle cells. It has been shown that UVA radiation can lead to an Immediate Pigment Darkening (IPD) response in melanocytes. Necessity of a protective response is obvious but this system feeds into a neuronal and hormonally-mediated centralised system of clock-like responses that not only respond to immediate threats but also demonstrating an established mechanism to anticipate changes in the day/night and seasonal cycles that have profound effects on the behaviour

Evolution has led us to our present complex level of behaviour but vestiges of our ancestral past linger in the complexity of responses to our environment. In the modern sophistication of our behaviour, with prolonged in-door living under artificial light, have we removed ourselves from the natural protective responses that eons of adaptation have provided for our bodies and left ourselves more open to solar-related malignancies

Sunlight has both beneficial and potentially carcinogenic effects. Unlike other mammals, humans lacking fur, are constantly exposed to the effects of Ultraviolet Radiation (UVR) in the out-door environment. Human skin requires protective mechanisms that can detect the threat and initiate an immediate response.

It has traditionally been accepted that all photoreception come through the eye. Photons are absorbed and converted to a cellular response. The photons interact with opsins (photosensitive proteins) via $\mathrm{G}$ protein-coupled opsin membrane receptors setting off a phototransduction signalling pathway. The eye has both image and non-image forming receptors which utilise different opsins and phototransduction cascades. The retina has rods and cone receptors for dim light and colour vision, respectively but some retinal ganglion cells utilise a different opsin, melanopsin, to initiate an immediate pupillary response to light but this also alerts the CNS to the presence of UVR. Beyond the potential threat, this response feeds into Circadian rhythm entrainment that has diverse and far reaching effects including sleep-wake cycles, feeding and reproductive responses. So, not only is a response initiated but the organism also needs a mechanism to be able to anticipate daily and seasonal changes in its requirements and responses to be prepared for, and survive, a constantly changing environment but with an element of periodic repetitive cycles.
There has found to be a retinal-dependent UV-sensitive phototransduction pathway in human melanocytes, similar to the non-visual receptors in the eye [1]. What is its significance and does it have a role in or beyond the traditionally accepted UVR-initiated keratinocyte DNA mutation, keratinocyte-melanocyte melanogenic pigmentary response?

\section{The Opsins}

Light is the ultimate energy source for most organisms and vitamin A-based chromophores have become the most widely used light sensor throughout evolution [2]. Light, however, is not just an energy source but also provides visual information and allows assessment of time. Opsins are proteins, in the form of 7 transmembrane $\alpha$-helices, bound to a vitamin A based chromophore (retinal) (Figure 1).

The chromophore, 11-Cis-retinal, with the absorption of a photon, isomerises to all-trans-retinal. There is a conformational change triggering a rearrangement of the photoreceptive protein structure causing a red shift from an absorbance max in the UV to the human visible range, matching the peak irradiance of sunlight on the earth's surface. This system is utilised in animals as light sensors for vision by transduction to an intracellular G-coupled membrane receptor. Unicellular organisms utilise light-gated channels, creating an electrochemical gradient to drive ATP synthase. The early evolutionary change to multi-cellular organism's G-coupled receptor was followed by a further evolutionary switch from bistable 


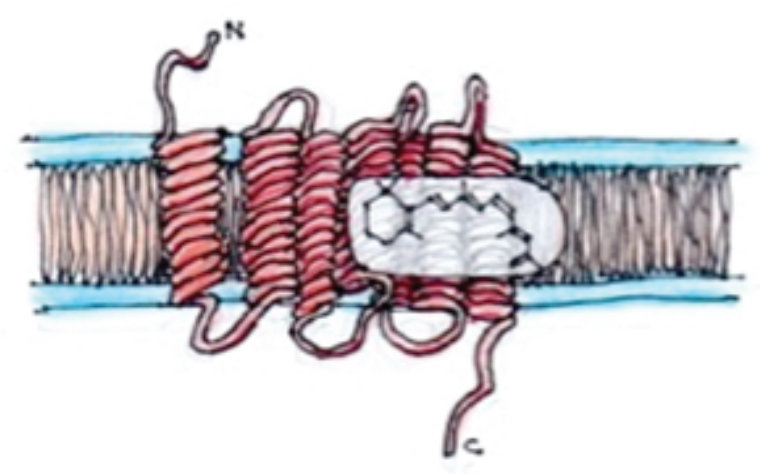

Figure 1: The structure of opsin: A representation of rhodopsin comprising an opsin protein with its 7 transmembrane $\alpha$-helices and a light-sensitive retinal chromophore binding to the $7^{\text {th }}$ transmembrane domain of the opsin. to monostable pigments despite the fact that monostable pigments rely on complex enzymatic cycles for regeneration after every photoisomerisation event, whereas the bistable pigmented only require light for regeneration. The bistable pigment exists as a mixture of two steady states, the 11-cis resting form and the all-trans excited form with the ability to regenerate the 11-cis retinal without the need for an isomerase. Longer wavelengths can change all-trans back into 11-cis retinal. The great advantage of the monostable pigments is that they can regenerate in the dark, providing more sensitive night vision. This permitted nocturnal mammals to forage at night avoiding competition with the dominant diurnal reptiles.

This also allowed an expansion of vitamin A biofunctions, including regulation of cell growth and differentiation at the expense of higher energy consumption and being more disease prone [2].

Some animal species have a large number of opsins and all species, including humans, have a range of opsins some with non-visual roles, and even light-independent roles.

The function of some of these opsins is still not understood. Possibly, rather than specifying them as photoreceptors, it would be more useful to consider them as a class of polymodal sensory receptors.

\section{Opsin expression in human skin}

Haltaufdehyde, et al. [3] found a range of opsins expressed in human epidermal keratinocytes and melanocytes. OPN1SW, OPN2, 3 and 5, with 2 and 3 most abundant (Figure 2). They found expression of opsin mRNA in skin and further suggested that these opsins might initiate light-induced signalling pathways, either singularly or in concert, possibly contributing to UVR phototransduction [3]. Wicks et al found that UVA induced a retinal dependent calcium flux mediated by a $\mathrm{G}$ protein coupled receptor in melanocytes, suggesting an opsin was involved. They went on to show that UVA phototransduction via OPN2, rhodopsin, and drives early melanin synthesis in melanocytes [4].

\section{Classification of Opsins}

Classification is based on the photoreceptor cell type that houses the photopigment: Ciliary (c-opsin) or rhabdomeric (r-opsin) [5]. Ciliary photo receptors, which include rods and cones, have stacked and flattened membranous discs. Rhabdomeric photoreceptors have

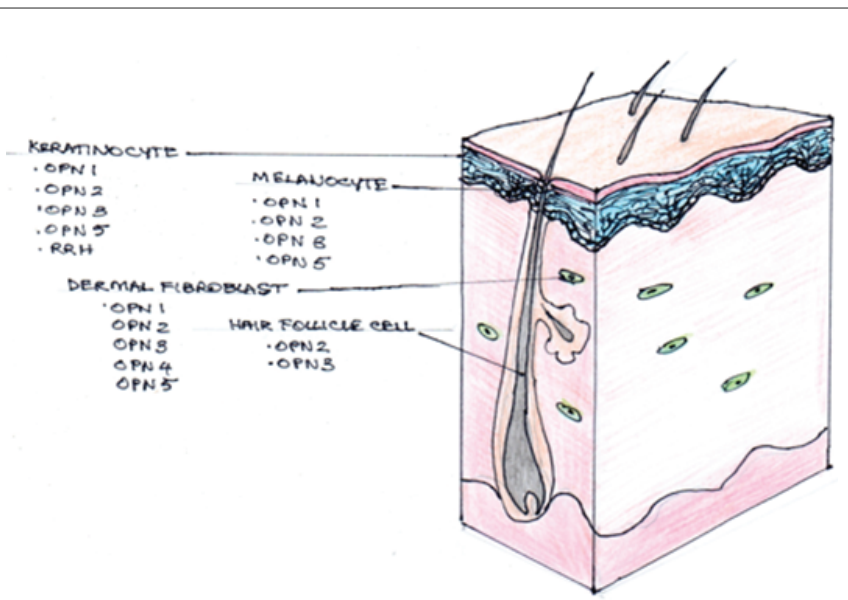

Figure 2: The expression of opsins in human skin cell types.

densely packed membranous microvilli projections, present in many invertebrates. The c-opsins and r-opsins couple to distinct signalling cascades that result in hypo- or depolarisation, respectively (Table 1).

It had already been established that UVB-induced DNA damage in keratinocytes led to transcriptional activation of melanogenic enzymes in melanocytes with delayed pigment darkening of the skin within days. UVA, however, causes oxidative damage with Immediate Pigment Darkening (IPD) within minutes, with increased melanin X5 within 24 hours. UVB pigment darkening requires de novo generation of tyrosinase, whereas, it has been suggested that the UVA response uses existing tyrosinase that becomes enzymatically active downstream of receptor activation [4].

Bellono, et al. demonstrated that human melanocytes use a UVR phototransduction mechanism that involves $\mathrm{Ga}_{\mathrm{q} / 11}$-dependent phosphoinositide cascade resembling light phototransduction in the eye. This requires GTP hydrolysis and phospholipase C $\beta$ (PC $\beta$ ) activating Transient Receptor Potential A1 (TRPA1) ion channels, increasing intracellular $\mathrm{Ca}^{2+}$ and subsequent cellular melanin content. The hydrolysis reaction also generates Diacylglycerol (DAG) and Inositol 1,4,5 triphosphate $\left(\mathrm{IP}_{3}\right)$ resulting in a rapid release of $\mathrm{Ca}^{2+}$ stores, adding to the influx through the ion channels (Figure 3) [1].

Unfortunately, however, de Assis, et al. [6] demonstrated that pigmentation generated during IPD does not absorb in the UV wavelength, so IPD does not protect DNA against the adverse effects of UVR [6]. Further recent studies demonstrated that IPD protects against deleterious effects of visible light, shown to penetrate deeply, reaching dermal capillaries [7]. Thus, IPD provides protection against degradation of folate derivatives and other chromophores in the visible light spectrum [8].

\section{The Molecular Clock}

In mammals, the Suprachiasmatic Nucleus (SCN) is the central oscillator responsible for controlling the temporal expression of clock genes and clock-controlled genes in peripheral tissues. The SCN has final control of the alignment of timing for the whole organism. The light and dark cycle is the timing clue (zeitgeber), the information interpreted by a subset of Retinal Ganglion Cells (ipRGCs) that express OPN4. The neuronal stimuli transmitted to the SCN through the retino-hypothalamic tract, which entrains the SCN and aligns 
Table 1: Classification of human opsins.

\begin{tabular}{|l|c|c|c|c|}
\hline $\begin{array}{l}\text { G } \\
\text { protein }\end{array}$ & Name & Ocular & Extraocular & $\begin{array}{c}\text { Absorptive } \\
\text { wavelength }\end{array}$ \\
\hline \multicolumn{5}{|c|}{ C-opsin (Ciliary) } \\
\hline$G_{t}$ & OPN 1-SW (Blue) & cones & Skin, sperm & $\sim 425$ \\
\hline$G_{t} G_{s}$ & OPN 1-MW (Green) & cones & Skin, sperm & $\sim 530$ \\
\hline$G_{t}$ & OPN 1-LW (Red) & cones & Skin & $\sim 560$ \\
\hline$G_{t}$ & OPN 2 Rhodopsin & rods & Skin, sperm & $\sim 500$ \\
\hline$G_{i} / G_{0}$ & OPN 3 Encephalopsin & Retina & sperm & $400-520$ \\
\hline \multicolumn{5}{|c|}{ R-opsin (Rhabdomeric) } \\
\hline$G_{q}$ & OPN 4 Melanopsin & $\begin{array}{c}\text { ipRGC, } \\
\text { Cones }\end{array}$ & $\begin{array}{c}\text { trigeminal } \\
\text { ganglion, } \\
\text { sperm }\end{array}$ & $\sim 480$ \\
\hline$G_{i}$ & OPN 5 Neuropsin & Retina & $\begin{array}{c}\text { Brain, spinal } \\
\text { cord, sperm, } \\
\text { testis }\end{array}$ & $\sim 380$ \\
\hline \multicolumn{7}{|c|}{ RG/G -opsins } \\
\hline
\end{tabular}

ipRGC: Intrinsically Photoreceptive Ganglion Cell; RGR: Retinal G-protein Coupled Receptor; RPE: Retinal Pigment Epithelium; RRH: Retinal Pigment Epithelial Derived Rhodopsin; ipRGCs: Intrinsically Photosensitive Retinal Ganglion Cells.
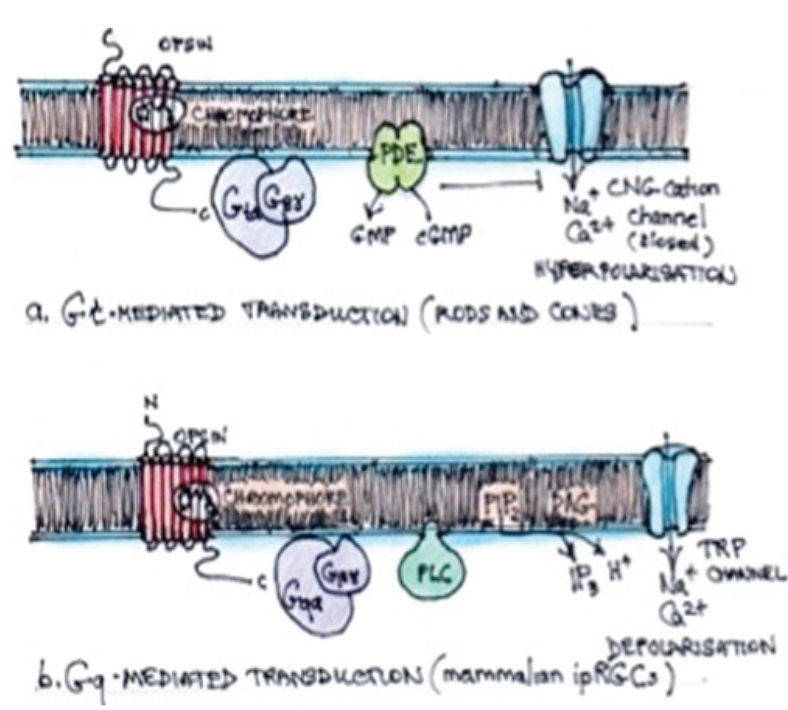

Figure 3: G protein-coupled transduction.

a). Gt mediated transduction couples an activated opsin to a Gt protein (transducin). Gt stimulates a Phosphodiesterase (PDE) causing a decline in cyclic GMP and subsequent closure of Cyclic NucleotideGated (CNG) cation channel, resulting in hyperpolarisation.

b). Gq mediated transduction couples an activated opsin to $\mathrm{Gq}$ which stimulates Phospholipase $C$ (PLC) resulting in hydrolysis of Phosphatidylinositol 4,5, Bisulphate (PIP2) opening TRP channels, resulting in depolarisation.

ipRGCs-intrinsically photosensitive retinal ganglion cells biological function to the circadian rhythm. The system consists of input from the ipRGCs, oscillatory machinery-SCN and peripheral oscillators located in most tissues and cells, and neural and hormonal output, including melatonin from the pineal gland (Figure 4).

Photons activate melanopsin expressing retinal ganglion cells which project through the optic nerve via the retino-hypothalamic tract to the Suprachiasmatic Nucleus (SCN). SCN neurons project to the paraventricular nuclei of the hypothalamus. There are further projections via preganglionic sympathetic neurons and postganglionic neurons in the superior cervical ganglia to the pineal gland.

Under natural conditions, circadian rhythms are entrained to the 24 hour light-dark cycle with daily rhythms of gene expression, body temperature, heart rate and melatonin production these rhythms affecting sleep, mood and cognitive function. The connection between circadian oscillators and health maintenance extends to the prevention and suppression of malignancies. Some core oscillator components having been shown to act as tumour suppressors $[9,10]$. A large proportion of the population showing some degree of chronodisruption in modern living.

Skin cells- keratinocytes, melanocytes and fibroblasts, display distinct clock gene machineries that comprise a local multi-oscillatory circadian system. Some skin functions have been found to oscillate in a circadian manner such as: blood flow, barrier function, transepidermal water loss, temperature and DNA repair. There is strong evidence that peripheral clocks can act as autonomous oscillators, receiving internal signals from the circadian master clock and external clues from the environment to locally coordinate cell and tissue functions and to maintain homeostasis [11].

\section{The Skin as a UV Sensor}

There has been a traditional view that Immediate Pigment Darkening (IPD) is UVB-induced oxidation of pre-existing melanin through keratinocyte DNA damage. Wicks et al, however, demonstrated that $\operatorname{UVR}\left(2 \mathrm{~kJ} / \mathrm{m}^{2}\right)$ induced changes in melanocytes involving OPN2 and a G-protein signalling cascade. Higher doses $\left(40 \mathrm{~kJ} / \mathrm{m}^{2}\right)$ increasing melanin levels with the suggestion that IPD is the result of de novo melanin synthesis. The calcium rise, mainly dependent on UVA and only an OPN2 transcript was detected in this study [4]. Further studies demonstrated the involvement of the Transient Receptor Potential Ankyrin 1 channel (TRPA1) responsible for calcium influx and the involvement of a specific G-protein, Gaq/11-dependent pathway. The combination of which led to a sustained rise in intracellular calcium levels resulting in melanin synthesis [1]. $\mathrm{Hu}$, et al. reported that both

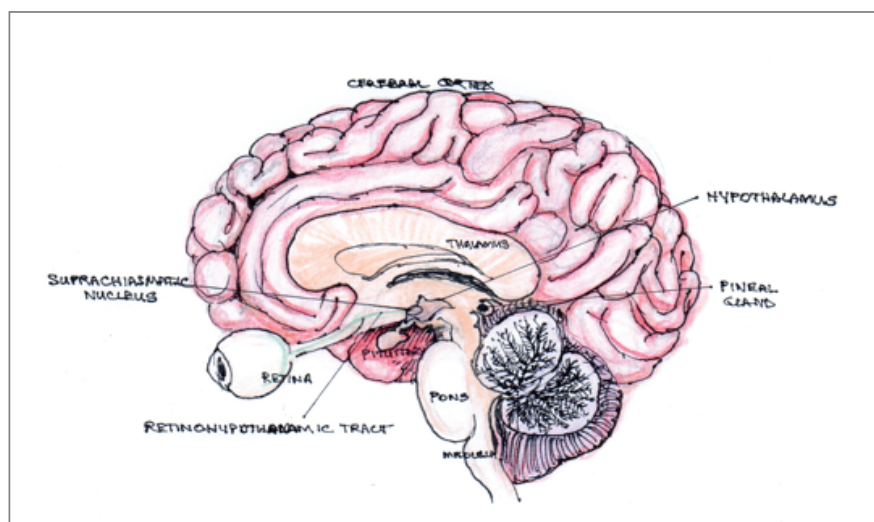

Figure 4: The retino-hypothalamo-pineal pathway. 
UVA and B elicited a calcium rise in melanocytes, only in the presence of retinal, leading to melanosome transfer but UVA was less effective at stimulation of melanin synthesis [12]. UVA and violet light were shown to increase OPN2 transcripts and down regulate keratinocyte differentiation markers [13]. Blue light on OPN3 is also possibly involved.

OPN5 has been demonstrated to be sensitive to both UVR and light with calcium rise, cAMP production and MAP activation [14]. So, UVR and visible light were influencing both melanocytes and keratinocytes through the opsin sensory system.

Castrucci's group demonstrated OPN 2 and 4 interact and are both necessary for UVA-induced IPD in murine normal and malignant melanocytes. They also demonstrated that OPN4 acts as a heat sensor and the UVA effect can be completely abolished at higher temperatures. This fact has been neglected in experimental design up till then [15].

UVA $\left(100 \mathrm{~kJ} / \mathrm{m}^{2}\right)$ increased OPN 1,3 and 5 protein levels without DNA damage in fibroblasts. Upon OPN3 knockdown the UVA-induced calcium rise was abolished along with matrix metalloproteinases, thus OPN3 was suggested as a factor in UVA-induced photoaging [16].

Most studies show involvement of OPN3 as a light/UVA sensor, however, Oancea's group found that it does not absorb in any UV or visible wavelength, thus, it is not a light sensor, despite its ability to bind to retinal. In fact, it is a negative regulator of melanogenesis in melanocytes, independent of UV or visible light, by inhibiting a melanocortin 1 receptor -dependent cAMP pathway [17]. OPN3, however, does have a light and temperature independent role. Knockdown led to apoptosis in a calcium-dependent manner with melanocyte mitochondrial permeability and caspase activation [16].

Castrucci's group also found UVA reduced proliferation in normal and malignant melanocytes, OPN4 expression decreasing with melanoma progression. Evidence of its involvement in UVA-induced apoptosis and melanoma pathogenesis [18].

\section{Can the Skin Sense Visible Light?}

Castrucci's group presented clear evidence of an opsin system (OPN2 and 4) operating in melanocytes and responding to visible light $\left(400-700 \mathrm{~nm}, 0.85 \mathrm{~kJ} / \mathrm{m}^{2}\right)$ in a murine model. Interestingly, malignant melanocytes were more sensitive to the effects than normal melanocytes. Melanin levels were, however, unaffected in both cell types [11].

It also appears that human hair follicles respond to blue light $\left(453 \mathrm{~nm}, 32 \mathrm{~kJ} / \mathrm{m}^{2}\right)$ with a prolonged anagen phase, involving OPN3 [19]. Violet light $\left(380 \mathrm{~nm}, 12 \mathrm{~kJ} / \mathrm{m}^{2}\right)$ induced a calcium flux in keratocytes possibly involving peropsin [20] and Buhr, et al. also found changes in OPN5 and circadian effects with violet light in mice [21]. So, multiple studies have demonstrated visible light effects lost with reduced opsin expression but this still does not necessarily mean that skin is sensing visible light.

Ozdeslik, et al. [3] reported that OPN3 is unable to absorb UV/ visible light photons suggesting that it participates in the signalling cascade without necessarily sensing light. OPN3 is also distributed in tissues that do not receive light, so having light-independent roles despite its ability to bind retinal [17]. Opsin have been shown to act as thermosensors and, in Drosophila, involved in taste discrimination [22], again arguing for opsins to be considered more as polymodal sensors and the need for cautious interpretation when investigators find changes in opsin expression as indicating a direct response to light.

\section{Conclusion}

The skin can be seen as a thing of beauty but this superficial assessment belies its activity as a sophisticated complex organ, having vital sensory and barrier functions for the organism as a whole. There needs to be constant monitoring of the surrounding environment and adjustment in a range of parameters for optimal homeostasis. In nonhuman animals, opsins are involved in behaviours that are critical for survival. Photoreception through dermal opsins enabling animals to instantly respond to changes of irradiation in local surroundings that may indicate potential danger. Moving themselves away to conceal themselves by camouflage or shadow reflex. Adjusting other movements and behaviours under the influence of circadian control.

The human has reached the apex of the food chain. The hunted becomes the hunter, and as such, humans live in a vastly different environment as compared to non-human animal species. The skin is the organ most extensively exposed to light over a broad range of wavelengths but now photoreception, visual and non-visual reception and imaging has evolved to be more concentrated at the head end within a complex visual apparatus having a close direct connection to the brain for central processing. Photoreception may now be seen as less vital through the skin allowing the opsins to be utilised as a more polymodal receptor. It is common feature through evolution for the peripheral system, and more particularly neural crest-derived cells, to co-opt molecular mechanisms and protein systems to new functions as adaption demands. It appears that opsins have biological functions beyond photoreception in human skin cells and cells in non-light exposed locations. They are not just vestigial evolutionary remnants from mechanisms developed in prehistory. So, despite irrefutable evidence of a photosensory system in the skin, involving opsins that respond to both UV and visible light, it is still not absolutely clear that this system directly responds to light transmitted through the skin.

Despite some remaining questions unanswered, can light, over various wavelengths, be used therapeutically? It appears that the response of melanocytes, keratinocytes, fibroblasts and hair follicle cells can be modified by specific wavelengths. Also, malignant melanocytes are more sensitive to these effects. So, not only may there be therapeutic application but there also needs to be an appreciation that the effect of modern life-style choices may be interrupting defence mechanisms built into circadian rhythms and internal biological clocks that responds to cycles of day/ night and seasonal changes that we appear to override and deny to our detriment by prolonged in-door living under artificial light.

\section{References}

1. Bellono NW, Najera JA, Oancea E (2014) UV light activates a Gaq/11coupled phototransduction pathway in human melanocytes. J Gen Physiol 143: 203-214.

2. Zhong M, Kawaguchi R, Kassai M, Sun H (2012) Retina, retinol, retinal and the natural history of vitamin $A$ as a light sensor. Nutrients 4 : 2069-2096.

3. Haltaufderhyde K, Ozdeslik RN, Wicks NL, Najera JA, Oancea E (2015) Opsin expression in human epidermal skin. Photochem Photobiol 91: 117-123.

4. Wicks NL, Chan JW, Najera JA, Ciriello JM, Oancea E (2011) UVA phototransduction drives early melanin synthesis in human melanocytes. Curr Biol 21: 1906-1911.

5. Yau KW, Hardie RC (2009) Phototransduction motifs and variations. Cell 139: 246-264. 
6. de Assis LVM, Moraes MN, Magalhães-Marques KK, de Lauro Castrucci AM (2018) Melanopsin and rhodopsin mediate UVA-induced immediate pigment darkening: Unravelling the photosensitive system of the skin. Eur J Cell Biol 97: 150-162.

7. Sklar LR, Almutawa F, Lim HW, Hamzavi I (2013) Effects of ultraviolet radiation, visible light, and infrared radiation on erythema and pigmentation: a review. Photochem Photobiol Sci 12: 54-64.

8. Moan J, Nielsen KP, Juzeniene A (2012) Immediate pigment darkening: its evolutionary roles may include protection against folate photosensitization. FASEB J 26: 971-975.

9. $\mathrm{Fu}$ L, Lee CC (2003) The circadian clock: pacemaker and tumour suppressor. Nat Rev Cancer 3: 350-361.

10. Yang X, Wood PA, Ansell CM, Quiton DFT, Oh EY, et al. (2009) The circadian clock gene Per1 suppresses cancer cell proliferation and tumor growth at specific times of day. Chronobiol Int 26: 1323-1339.

11. de Assis LVM, Moraes MN, da Silveira Cruz-Machado S, Castrucci AML (2016) The effect of white light on normal and malignant murine melanocytes: A link between opsins, clock genes, and melanogenesis. Biochim Biophys Acta 1863: 1119-1133.

12. Hu QM, Yi WJ, Su MY, Jiang S, Xu SH, et al. (2017) Induction of retinaldependent calcium influx in human melanocytes by UVA or UVB radiation contributes to the stimulation of melanosome transfer. Cell Prolif 50: e12372.

13. Slominski AT, Zmijewski MA, Skobowiat C, Zbytek B, Slominski RM, et al. (2012) Sensing the environment: regulation of local and global homeostasis by the skin's neuroendocrine system. Adv Anat Embryol Cell Biol 212; 1-115.

14. Sugiyama T, Suzuki H, Takahashi T (2014) Light-induced rapid $\mathrm{Ca}^{2+}$ response and MAPK phosphorylation in the cells heterologously expressing human OPN5. Sci Rep 4: 5352.
15. De Assis L, Moraes M, Castrucci A (2017) Heat shock antagonizes UVA-induced responses in murine melanocytes and malignant cells: an unexpected interaction. Photochem Photobiol 16: 633-648.

16. Wang Y, Lan Y, Lu H (2020) Opsin3 Downregulation Induces Apoptosis of Human Epidermal Melanocytes via Mitochondrial Pathway. Photochem Photobiol 96: 83-93.

17. Ozdeslik RN, Olinski LE, Trieu MM, Oprian DD, Oancea E (2019) Human nonvisual opsin 3 regulates pigmentation of epidermal melanocytes through functional interaction with melanocortin 1 receptor. PNAS 116: 11508-11517

18. de Assis LVM, Mendes D, Silva MM, Kinker GS, Pereira-Lima I, et al. (2020) Melanopsin mediates UVA-dependent modulation of proliferation, pigmentation, apoptosis, and molecular clock in normal and malignant melanocytes. Biochim Biophys Acta Mol Cell Res 1867: 118789.

19. Buscone S, Mardaryev AN, Raafs B, Bikker JW, Sticht C, et al. (2017) A new path in defining light parameters for hair growth: Discovery and modulation of photoreceptors in human hair follicle. Lasers Surg Med 49: 705-718.

20. Toh PPC, Bigliardi-Qi M, Yap AMY, Sriram G, Stelmashenko O, et al. (2016) Expression of peropsin in human skin is related to phototransduction of violet light in keratinocytes. Exp Dermatol 25: 1002-1005

21. Buhr ED, Vemaraju S, Diaz N, Lang RA, Gelder RNV (2019) Neuropsin (OPN5) Mediates Local Light-Dependent Induction of Circadian Clock Genes and Circadian Photoentrainment in Exposed Murine Skin. Current Biol 29: 3478-3487.

22. Leung NY, Thakur DP, Gurav AS, Kim SH, Pizio AD, et al. (2020) Functions of Opsins in Drosophila Taste. Curr Biol 30: 1367-1379. 